\title{
On the Periodic Orbits of Four-Particle Time-Dependent FPU Chains
}

\author{
Hongwu Tang, ${ }^{1,2}$ Mingyan Xing, ${ }^{2}$ Qihuai Liu $\mathbb{D}^{2},{ }^{2}$ and Guirong Jiang ${ }^{2}$ \\ ${ }^{1}$ School of Mathematics and Statistics, Central South University, Changsha 410083, China \\ ${ }^{2}$ School of Mathematics and Computing Sciences, Guangxi Colleges and Universities Key Laboratory of \\ Data Analysis and Computation, Guilin University of Electronic Technology, Guilin 541002, China \\ Correspondence should be addressed to Qihuai Liu; qhuailiu@gmail.com
}

Received 4 December 2017; Accepted 15 March 2018; Published 22 April 2018

Academic Editor: Zhijun Qiao

Copyright (C) 2018 Hongwu Tang et al. This is an open access article distributed under the Creative Commons Attribution License, which permits unrestricted use, distribution, and reproduction in any medium, provided the original work is properly cited.

We study the existence of the periodic orbits for four-particle time-dependent FPU chains. To begin with, taking an orthogonal transformation and dropping two variables, we put the system into a lower dimensional system. In this case, the unperturbed system has the linear spectra 2 and $\sqrt{2}$, which are rational independent. Therefore, the classical averaging theory cannot be applied directly, and the existence and multiplicity of periodic orbits for four-particle FPU chains is proved by using a new result from averaging theory by Buică, Francoise, and Llibre.

\section{Introduction}

The Fermi-Pasta-Ulam (FPU) system describes an approximate model for the behaviour of a classical solid at low temperatures [1], whose development dates from the first numerical results of FPU by Fermi et al. in 1955 [2]. FPU problem, both ushering in the age of computational science and marking the birth of our own field of nonlinear science [3], has received the widespread attention both in mathematics and in physics, including ergodicity $[4,5]$, integrability $[6,7]$, breathers [8-10], chaos, and stability of motion [11-14]. In fact, the FPU problem is much more than a little discovery and will doubtless be challenging scientists and stimulating new research directions for many decades to come [3].

In this paper, we consider the time-dependent Hamiltonian:

$$
H(t, q, p)=\sum_{j \in \mathbb{Z} / n \mathbb{Z}} \frac{1}{2} p_{j}^{2}+W\left(t, q_{j+1}-q_{j}\right),
$$

where $q_{j}$ denotes the displacement of the $j$ th oscillator with respect to its equilibrium position. The coupled equations of motions are

$$
\begin{array}{r}
\ddot{q}_{j}+W_{q}^{\prime}\left(t, q_{j}-q_{j-1}\right)-W_{q}^{\prime}\left(t, q_{j+1}-q_{j}\right)=0, \\
q_{j}=q_{j+n}, n \in \mathbb{N}, j \in \mathbb{Z},
\end{array}
$$

and $W(t, q)$, denoting the coupling potential between the neighboring particles, is given by

$$
\begin{aligned}
W(t, q)= & \frac{1}{2} q^{2}+\frac{\alpha(t)}{3} q^{3}+\frac{\beta(t)}{4} q^{4}+\frac{\chi(t)}{5} q^{5} \\
& +\frac{\gamma(t)}{6} q^{6}+\text { h.o.t.. }
\end{aligned}
$$

Here, h.o.t. is the high order term of $q^{6}$, and $\alpha(t), \beta(t), \chi(t)$, $\gamma(t)$ are continuous $T$-periodic functions. If $\beta \equiv 0, \chi \equiv 0$, $\gamma \equiv 0, \alpha \neq 0$, the chain is called an $\alpha$-chain, and if $\alpha \equiv 0$, $\chi \equiv 0, \gamma \equiv 0, \beta \neq 0$, it is called a $\beta$-chain. The FPU model with the time-dependent nonlinear term is used to explore the relation between the thermodynamic entropy change due to irreversible processes and the change of information loss as a chaotic dynamical system [15]. For a recent report, we can refer to [16].

In low frequency modes and the transfer of energy to higher modes, periodic orbits of FPU lattices have been widely studied [17, 18]. In [18], Flach and Ponno discussed the FPU problem including periodic orbits, normal forms, and resonance overlap criteria, compared all these results with each other, and established the similarities and differences. The paper [19] studied weakly nonlinear spatially localized solutions of a FPU model which describe a one-dimensional 
chain of particles interacting with a number of neighbors depended on the site. When $W(t, q)$ is asymptotically linear with respect to $q$ both at origin and at infinity, the existence of nontrivial periodic solutions of system (2) has been proved in [20]. In this paper, we study existence of periodic orbits for four-particle time-dependent FPU chains by using the averaging theory.

The averaging method, an important method of perturbation theory that has played a rather modest role in nonlinear differential system with a small parameter, gives useful approximate solutions of the equations of motion [21]. For instance, the existence of periodic orbits and the stability of system have been studied using averaging method in [22-26]. In the previous work [27], we have studied the existence of periodic orbits and their stability by using the classical averaging method for the three-particle timedependent FPU chains, where the system can be regarded as a perturbation of isochronous system. In this paper, we shall investigate the existence and multiplicity of periodic orbits for four-particle time-dependent FPU chains. In this case, the unperturbed system has the linear spectra 2 and $\sqrt{2}$, which are rational independent. Therefore, the classical averaging theory cannot be applied directly. For some specific coefficients being usually used in the physical experiment, we establish the existence and multiplicity of periodic orbits for four-particle time-dependent FPU chains by using the result from Buică et al. [28].

The rest of paper is organized as follows. In Section 2, we present our main results on the periodic solutions of the FPU chains and introduce the basic results from averaging theory in Section 3. The detailed proofs for the existence and multiplicity of periodic orbits of four-particle timedependent FPU chains are given in the last part.

\section{Statement of the Results}

Consider the time-dependent Hamiltonian:

$$
\begin{aligned}
H(t, q, p)= & \sum_{j \in \mathbb{Z} / 4 \mathbb{Z}} \frac{1}{2} p_{j}^{2}+\frac{1}{2}\left(q_{j+1}-q_{j}\right)^{2} \\
& +\epsilon \frac{\alpha(t)}{3}\left(q_{j+1}-q_{j}\right)^{3} \\
& +\epsilon \frac{\beta(t)}{4}\left(q_{j+1}-q_{j}\right)^{4} \\
& +\epsilon \frac{\chi(t)}{5}\left(q_{j+1}-q_{j}\right)^{5} \\
& +\epsilon \frac{\gamma(t)}{6}\left(q_{j+1}-q_{j}\right)^{6}+\mathcal{O}\left(\epsilon^{2}\right),
\end{aligned}
$$

where $q_{j+4}=q_{j}, p_{j+4}=p_{j}, j \in \mathbb{Z}, \epsilon$ is a small parameter introduced by a scale transformation and $\alpha, \beta, \chi, \gamma$ are $T$-periodic $C^{2}$ smoothness functions. The corresponding Hamiltonian system is given by

$$
\dot{q}=p
$$

$$
\begin{aligned}
\dot{p}= & -A q-\epsilon \alpha(t) B_{1}(t, q)-\epsilon \beta(t) B_{2}(t, q) \\
& -\epsilon \chi(t) B_{3}(t, q)-\epsilon \gamma(t) B_{4}(t, q)+\mathcal{O}\left(\epsilon^{2}\right),
\end{aligned}
$$

where $B_{k}(t, q)$ is defined by

$$
B_{k}(t, q)=\left[\begin{array}{l}
\left(q_{1}-q_{4}\right)^{k+1}-\left(q_{2}-q_{1}\right)^{k+1} \\
\left(q_{2}-q_{1}\right)^{k+1}-\left(q_{3}-q_{2}\right)^{k+1} \\
\left(q_{3}-q_{2}\right)^{k+1}-\left(q_{4}-q_{3}\right)^{k+1} \\
\left(q_{4}-q_{3}\right)^{k+1}-\left(q_{1}-q_{4}\right)^{k+1}
\end{array}\right], \quad
$$

with $q=\left(q_{1}, \ldots, q_{4}\right)^{T}, p=\left(p_{1}, \ldots, p_{4}\right)^{T} \in \mathbb{R}^{4}$, and $A$ is a real symmetric and positive semidefinite matrix, which is given by

$$
A=\left(\begin{array}{cccc}
2 & -1 & 0 & -1 \\
-1 & 2 & -1 & 0 \\
0 & -1 & 2 & -1 \\
-1 & 0 & -1 & 2
\end{array}\right) .
$$

Taking an orthogonal transformation by

$$
\begin{aligned}
& \bar{q}=\left(\begin{array}{cccc}
-\frac{1}{2} & \frac{1}{2} & -\frac{1}{2} & \frac{1}{2} \\
0 & -\frac{1}{\sqrt{2}} & 0 & \frac{1}{\sqrt{2}} \\
-\frac{1}{\sqrt{2}} & 0 & \frac{1}{\sqrt{2}} & 0 \\
\frac{1}{2} & \frac{1}{2} & \frac{1}{2} & \frac{1}{2}
\end{array}\right) q, \\
& \bar{p}=\left(\begin{array}{cccc}
-\frac{1}{2} & \frac{1}{2} & -\frac{1}{2} & \frac{1}{2} \\
0 & -\frac{1}{\sqrt{2}} & 0 & \frac{1}{\sqrt{2}} \\
-\frac{1}{\sqrt{2}} & 0 & \frac{1}{\sqrt{2}} & 0 \\
\frac{1}{2} & \frac{1}{2} & \frac{1}{2} & \frac{1}{2}
\end{array}\right) p,
\end{aligned}
$$

system (5) transforms into

$$
\begin{aligned}
& \dot{\bar{q}}_{1}=\bar{p}_{1}, \\
& \dot{\bar{q}}_{2}=\bar{p}_{2}, \\
& \dot{\bar{q}}_{3}=\bar{p}_{3}, \\
& \dot{\bar{q}}_{4}=\bar{p}_{4}, \\
& \dot{\bar{p}}_{1}=-4 \bar{q}_{1}+\epsilon F_{1}(t, \bar{q}, \bar{p})+\mathcal{O}\left(\epsilon^{2}\right), \\
& \dot{\bar{p}}_{2}=-2 \bar{q}_{2}+\epsilon F_{2}(t, \bar{q}, \bar{p})+\mathcal{O}\left(\epsilon^{2}\right), \\
& \dot{\bar{p}}_{3}=-2 \bar{q}_{3}+\epsilon F_{3}(t, \bar{q}, \bar{p})+\mathcal{O}\left(\epsilon^{2}\right), \\
& \dot{\bar{p}}_{4}=0,
\end{aligned}
$$


where

$$
\begin{aligned}
& F_{1}(t, \bar{q}, \bar{p})=4 \bar{q}_{2} \bar{q}_{3}\left(\alpha+\chi\left(6 \bar{q}_{1}^{2}+\bar{q}_{2}^{2}+\bar{q}_{3}^{2}\right)\right) \\
& \quad-2 \beta \bar{q}_{1}\left(2 \bar{q}_{1}^{2}+3 \bar{q}_{2}^{2}+3 \bar{q}_{3}^{2}\right)-\gamma \bar{q}_{1}\left(4 \bar{q}_{1}^{4}+5 \bar{q}_{2}^{4}+5 \bar{q}_{3}^{4}\right. \\
& \left.\quad+10\left(2 \bar{q}_{1}^{2} \bar{q}_{2}^{2}+2 \bar{q}_{1}^{2} \bar{q}_{3}^{2}+3 \bar{q}_{2}^{2} \bar{q}_{3}^{2}\right)\right), \\
& F_{2}(t, \bar{q}, \bar{p})=4 \bar{q}_{1} \bar{q}_{3}\left(\alpha+\chi\left(2 \bar{q}_{1}^{2}+3 \bar{q}_{2}^{2}+\bar{q}_{3}^{2}\right)\right) \\
& \quad-\beta \bar{q}_{2}\left(6 \bar{q}_{1}^{2}+\bar{q}_{2}^{2}+3 \bar{q}_{3}^{2}\right)-\frac{1}{2} \gamma \bar{q}_{2}\left(20 \bar{q}_{1}^{4}+\bar{q}_{2}^{4}+5 \bar{q}_{3}^{4}\right. \\
& \left.\quad+10\left(2 \bar{q}_{1}^{2} \bar{q}_{2}^{2}+6 \bar{q}_{1}^{2} \bar{q}_{3}^{2}+\bar{q}_{2}^{2} \bar{q}_{3}^{2}\right)\right), \\
& F_{3}(t, \bar{q}, \bar{p})=4 \bar{q}_{1} \bar{q}_{2}\left(\alpha+\chi\left(2 \bar{q}_{1}^{2}+\bar{q}_{2}^{2}+3 \bar{q}_{3}^{2}\right)\right) \\
& \quad-\beta \bar{q}_{3}\left(6 \bar{q}_{1}^{2}+3 \bar{q}_{2}^{2}+\bar{q}_{3}^{2}\right)-\frac{1}{2} \gamma \bar{q}_{3}\left(20 \bar{q}_{1}^{4}+5 \bar{q}_{2}^{4}+\bar{q}_{3}^{4}\right. \\
& \left.\quad+10\left(6 \bar{q}_{1}^{2} \bar{q}_{2}^{2}+2 \bar{q}_{3}^{2} \bar{q}_{1}^{2}+\bar{q}_{2}^{2} \bar{q}_{3}^{2}\right)\right) .
\end{aligned}
$$

In system (9), we drop equations of $\bar{q}_{4}, \bar{p}_{4}$ in the subsequent discussion since these equations are integrable. Only when $\bar{q}_{4}, \bar{p}_{4}$ are equal to zero, system (9) has periodic solutions. Therefore, system (9) can be expressed as

$$
\begin{aligned}
& \dot{\bar{q}}_{1}=\bar{p}_{1}, \\
& \dot{\bar{q}}_{2}=\bar{p}_{2}, \\
& \dot{\bar{q}}_{3}=\bar{p}_{3}, \\
& \dot{\bar{p}}_{1}=-4 \bar{q}_{1}+\epsilon F_{1}(t, \bar{q}, \bar{p})+\mathcal{O}\left(\epsilon^{2}\right), \\
& \dot{\bar{p}}_{2}=-2 \bar{q}_{2}+\epsilon F_{2}(t, \bar{q}, \bar{p})+\mathcal{O}\left(\epsilon^{2}\right), \\
& \dot{\bar{p}}_{3}=-2 \bar{q}_{3}+\epsilon F_{3}(t, \bar{q}, \bar{p})+\mathcal{O}\left(\epsilon^{2}\right) .
\end{aligned}
$$

The unperturbed system of (11) with $\epsilon=0$ is given by

$$
\begin{aligned}
& \dot{\bar{q}}_{1}=\bar{p}_{1}, \\
& \dot{\bar{q}}_{2}=\bar{p}_{2}, \\
& \dot{\bar{q}}_{3}=\bar{p}_{3} \\
& \dot{\bar{p}}_{1}=-4 \bar{q}_{1}, \\
& \dot{\bar{p}}_{2}=-2 \bar{q}_{2}, \\
& \dot{\bar{p}}_{3}=-2 \bar{q}_{3},
\end{aligned}
$$

which has a rest point at the origin with eigenvalues $\pm 2 i$, $\pm \sqrt{2} i$, these last two with multiplicity two. Consequently, this system in the phase space $\left(\bar{q}_{1}, \bar{p}_{1}, \bar{q}_{2}, \bar{p}_{2}, \bar{q}_{3}, \bar{p}_{3}\right)$ has three planes filled with periodic solutions with the exception of the origin. These periodic solutions have periods $T_{1}=\pi$ or $T_{2}=$ $\sqrt{2} \pi$, according to whether they belong to the plane associated with the eigenvectors with eigenvalues $\pm 2 i$ or $\pm \sqrt{2} i$. In this paper, we shall study which of these periodic solutions persist for the perturbed system (11) thereby for FPU system (5), when the parameter $\epsilon$ is sufficiently small and the perturbed functions $F_{i}$ for $i=1,2,3$ have period either $T_{1}$ or $T_{2}$.

The solution of unperturbed system (12) with initial value

$$
\begin{aligned}
& \left(\bar{p}_{1}(0), \bar{q}_{1}(0), \bar{p}_{2}(0), \bar{q}_{2}(0), \bar{p}_{3}(0), \bar{q}_{3}(0)\right) \\
& \quad=\left(\bar{p}_{10}, \bar{q}_{10}, \bar{p}_{20}, \bar{q}_{20}, \bar{p}_{30}, \bar{q}_{30}\right)
\end{aligned}
$$

is given by

$$
\begin{aligned}
& \bar{q}_{1}(t)=\bar{q}_{10} \cos (2 t)+\frac{1}{2} \bar{p}_{10} \sin (2 t), \\
& \bar{p}_{1}(t)=\bar{p}_{10} \cos (2 t)-2 \bar{q}_{10} \sin (2 t), \\
& \bar{q}_{2}(t)=\bar{q}_{20} \cos (\sqrt{2} t)+\frac{1}{\sqrt{2}} \bar{p}_{20} \sin (\sqrt{2} t), \\
& \bar{p}_{2}(t)=\bar{p}_{20} \cos (\sqrt{2} t)-\sqrt{2} \bar{q}_{20} \sin (\sqrt{2} t), \\
& \bar{q}_{3}(t)=\bar{q}_{30} \cos (\sqrt{2} t)+\frac{1}{\sqrt{2}} \bar{p}_{30} \sin (\sqrt{2} t), \\
& \bar{p}_{3}(t)=\bar{p}_{30} \cos (\sqrt{2} t)-\sqrt{2} \bar{q}_{30} \sin (\sqrt{2} t) .
\end{aligned}
$$

We define the functions as follows:

$$
\begin{aligned}
& \mathscr{F}_{1}\left(\bar{q}_{10}, \bar{p}_{10}\right)=-\int_{0}^{T_{1}} \frac{\sin (2 t)}{2} F_{1}\left(t, \bar{q}_{1}(t), \bar{p}_{1}(t), 0,0,\right. \\
& 0,0) \mathrm{d} t, \\
& \mathscr{F}_{2}\left(\bar{q}_{10}, \bar{p}_{10}\right)=\int_{0}^{T_{1}} \cos (2 t) F_{1}\left(t, \bar{q}_{1}(t), \bar{p}_{1}(t), 0,0,\right. \\
& 0,0) \mathrm{d} t, \\
& \mathscr{G}_{1}\left(\bar{q}_{20}, \bar{p}_{20}, \bar{q}_{30}, \bar{p}_{30}\right)=-\int_{0}^{T_{2}} \frac{\sin (\sqrt{2} t)}{\sqrt{2}} F_{2}(t, 0,0, \\
& \left.\bar{q}_{2}(t), \bar{p}_{2}(t), \bar{q}_{3}(t), \bar{p}_{3}(t)\right) \mathrm{d} t, \\
& \mathscr{G}_{2}\left(\bar{q}_{20}, \bar{p}_{20}, \bar{q}_{30}, \bar{p}_{30}\right)=\int_{0}^{T_{2}} \cos (\sqrt{2} t) F_{2}\left(t, 0,0, \bar{q}_{2}(t),\right. \\
& \left.\bar{p}_{2}(t), \bar{q}_{3}(t), \bar{p}_{3}(t)\right) \mathrm{d} t, \\
& \mathscr{G}_{3}\left(\bar{q}_{20}, \bar{p}_{20}, \bar{q}_{30}, \bar{p}_{30}\right)=-\int_{0}^{T_{2}} \frac{\sin (\sqrt{2} t)}{\sqrt{2}} F_{3}(t, 0,0, \\
& \left.\bar{q}_{2}(t), \bar{p}_{2}(t), \bar{q}_{3}(t), \bar{p}_{3}(t)\right) \mathrm{d} t, \\
& \mathscr{G}_{4}\left(\bar{q}_{20}, \bar{p}_{20}, \bar{q}_{30}, \bar{p}_{30}\right)=\int_{0}^{T_{2}} \cos (\sqrt{2} t) F_{3}\left(t, 0,0, \bar{q}_{2}(t),\right. \\
& \left.\bar{p}_{2}(t), \bar{q}_{3}(t), \bar{p}_{3}(t)\right) \mathrm{d} t .
\end{aligned}
$$

A solution $\left(q_{10}^{*}, p_{10}^{*}\right)$ of the algebraic equations

$$
\begin{aligned}
& \mathscr{F}_{1}\left(\bar{q}_{10}, \bar{p}_{10}\right)=0, \\
& \mathscr{F}_{2}\left(\bar{q}_{10}, \bar{p}_{10}\right)=0,
\end{aligned}
$$


such that

$$
\operatorname{det}\left(\left.\frac{\partial\left(\mathscr{F}_{1}, \mathscr{F}_{2}\right)}{\partial\left(\bar{q}_{10}, \bar{p}_{10}\right)}\right|_{\left(\bar{q}_{10}, \bar{p}_{10}\right)=\left(q_{10}^{*}, p_{10}^{*}\right)}\right) \neq 0,
$$

is called a simple zero of the averaged system associated with (11). Similarly, a solution $\left(\bar{q}_{20}, \bar{p}_{20}, \bar{q}_{30}, \bar{p}_{30}\right)$ of the algebraic equations

$$
\mathscr{G}_{i}\left(\bar{q}_{20}, \bar{p}_{20}, \bar{q}_{30}, \bar{p}_{30}\right)=0, \quad i=1,2,3,4,
$$

such that

$$
\begin{aligned}
& \operatorname{det}\left(\left.\frac{\partial\left(\mathscr{G}_{1}, \mathscr{G}_{2}, \mathscr{G}_{3}, \mathscr{G}_{4}\right)}{\partial\left(\bar{q}_{20}, \bar{p}_{20}, \bar{q}_{30}, \bar{p}_{30}\right)}\right|_{\left(\bar{q}_{20}, \bar{p}_{20}, \bar{q}_{30}, \bar{p}_{30}\right)=\left(q_{20}^{*}, p_{20}^{*}, q_{30}^{*}, p_{30}^{*}\right)}\right) \\
& \quad \neq 0
\end{aligned}
$$

is also called a simple zero of the averaged system associated with (11).

Since scale transformation is used to the change of variables for all $\epsilon>0$, the transformed system (9) and the original system (5) have the same topological structure. In the following, we state our main results.

Theorem 1. Assume that $\beta(t)=a+b \cos (4 t), \gamma(t)=\gamma_{0}, a, b$, $\gamma_{0} \in \mathbb{R}, b \neq 0$ and $\alpha(t), \chi(t)$ are $C^{2}$ periodic functions with the same period $\pi$. Then, for $\varepsilon \neq 0$ sufficiently small, the following statements hold:

(a) if $(3 a+2 b) \gamma_{0}<0$, system (5) has at least two $\pi$-periodic solutions,

$$
\begin{aligned}
& \left(q_{1}(t, \epsilon), p_{1}(t, \epsilon), q_{2}(t, \epsilon), p_{2}(t, \epsilon), q_{3}(t, \epsilon), p_{3}(t, \epsilon),\right. \\
& \left.\quad q_{4}(t, \epsilon), p_{4}(t, \epsilon)\right)
\end{aligned}
$$

such that

$\lim _{\epsilon \rightarrow 0}\left(q_{1}(0, \epsilon), p_{1}(0, \epsilon), q_{2}(0, \epsilon), p_{2}(0, \epsilon), q_{3}(0, \epsilon)\right.$,

$$
\begin{aligned}
& \left.p_{3}(0, \epsilon), q_{4}(0, \epsilon), p_{4}(0, \epsilon)\right)=\left(\mp \sqrt{\frac{-(3 a+2 b)}{10 \gamma_{0}}}, 0,\right. \\
& \pm \sqrt{\frac{-(3 a+2 b)}{10 \gamma_{0}}}, 0, \mp \sqrt{\frac{-(3 a+2 b)}{10 \gamma_{0}}}, 0, \\
& \left. \pm \sqrt{\frac{-(3 a+2 b)}{10 \gamma_{0}}}, 0\right) .
\end{aligned}
$$

(b) if $(2 b-3 a) \gamma_{0}>0$, system (5) has at least one $\pi$-periodic solutions,

$$
\begin{aligned}
& \left(q_{1}(t, \epsilon), p_{1}(t, \epsilon), q_{2}(t, \epsilon), p_{2}(t, \epsilon), q_{3}(t, \epsilon), p_{3}(t, \epsilon),\right. \\
& \left.q_{4}(t, \epsilon), p_{4}(t, \epsilon)\right)
\end{aligned}
$$

such that

$$
\begin{aligned}
& \lim _{\epsilon \rightarrow 0}\left(q_{1}(0, \epsilon), p_{1}(0, \epsilon), q_{2}(0, \epsilon), p_{2}(0, \epsilon), q_{3}(0, \epsilon),\right. \\
& \left.p_{3}(0, \epsilon), q_{4}(0, \epsilon), p_{4}(0, \epsilon)\right)=\left(0, \mp \sqrt{\frac{2(2 b-3 a)}{5 \gamma_{0}}},\right. \\
& 0, \pm \sqrt{\frac{2(2 b-3 a)}{5 \gamma_{0}}}, 0, \mp \sqrt{\frac{2(2 b-3 a)}{5 \gamma_{0}}}, 0, \\
& \left. \pm \sqrt{\frac{2(2 b-3 a)}{5 \gamma_{0}}}\right) .
\end{aligned}
$$

Theorem 2. Assume that $\beta(t)=b \cos (2 \sqrt{2} t), \gamma(t)=\gamma_{0}$, $b, \gamma_{0} \in \mathbb{R}$, and the functions $\alpha(t), \chi(t)$ are of class $C^{2}$ and $\sqrt{2} \pi$ periodic. For $\varepsilon \neq 0$ sufficiently small, if $b \gamma_{0}>0$, system (5) has at least four $\sqrt{2} \pi$-periodic solutions:

$$
\begin{gathered}
\left(q_{1}(t, \epsilon), p_{1}(t, \epsilon), q_{2}(t, \epsilon), p_{2}(t, \epsilon), q_{3}(t, \epsilon), p_{3}(t, \epsilon),\right. \\
\left.q_{4}(t, \epsilon), p_{4}(t, \epsilon)\right)
\end{gathered}
$$

such that

$$
\begin{gathered}
\lim _{\epsilon \rightarrow 0}\left(q_{1}(0, \epsilon), p_{1}(0, \epsilon), q_{2}(0, \epsilon), p_{2}(0, \epsilon), q_{3}(0, \epsilon),\right. \\
\left.p_{3}(0, \epsilon), q_{4}(0, \epsilon), p_{4}(0, \epsilon)\right)=\left(0,0,0, \mp 2 \sqrt{\frac{2 b}{5 \gamma_{0}}},\right. \\
\left.0,0,0, \pm 2 \sqrt{\frac{2 b}{5 \gamma_{0}}}\right),
\end{gathered}
$$

or

$$
\lim _{\epsilon \rightarrow 0}\left(q_{1}(0, \epsilon), p_{1}(0, \epsilon), q_{2}(0, \epsilon), p_{2}(0, \epsilon), q_{3}(0, \epsilon),\right.
$$

$$
\begin{aligned}
& \left.p_{3}(0, \epsilon), q_{4}(0, \epsilon), p_{4}(0, \epsilon)\right) \\
& =\left(0, \mp 2 \sqrt{\frac{2 b}{5 \gamma_{0}}}, 0,0,0, \pm 2 \sqrt{\frac{2 b}{5 \gamma_{0}}}, 0,0\right) .
\end{aligned}
$$

\section{Some Results from Averaging Theory}

In this section, we present the basic result from the averaging theory that we apply to prove our main results.

We consider the problem of the bifurcation of $T$-periodic solutions from differential systems of the following form:

$$
\dot{X}(t)=G_{0}(t, X)+\varepsilon G_{1}(t, X)+\varepsilon^{2} G_{2}(t, X)
$$

with $\varepsilon \neq 0$ being sufficiently small, where $G_{0}, G_{1}: \mathbb{R} \times \Omega \rightarrow$ $\mathbb{R}^{n}$ and $G_{2}: \mathbb{R} \times \Omega \times\left(-\varepsilon_{0}, \varepsilon_{0}\right) \rightarrow \mathbb{R}^{n}$ are $C^{2}$ smoothness and $T$-periodic in $t$, and $\Omega$ is an open subset of $\mathbb{R}^{n}$. When $\varepsilon=0$, the unperturbed system is given by

$$
\dot{X}(t)=G_{0}(t, X),
$$


and we assume that it has a submanifold of periodic solutions. Using the classical averaging theory, the existence of $T$ periodic solutions of system (27) can be established with nondegenerate equilibrium points of the associated averaged system. We can refer to [29] for details.

Denote the linearization of the unperturbed system (28) along $X(t, z)$ by

$$
\dot{Y}(t)=D_{X} G_{0}(t, X(t, z)) Y,
$$

where $X(t, z)$ is the periodic solution of the system (28) such that $X(0, z)=z$. For simplicity, we assume that $M_{z}(t)$ is a fundamental matrix of the linear differential system (29), and $\xi$ : $\mathbb{R}^{k} \times \mathbb{R}^{n-k} \rightarrow \mathbb{R}^{k}$, is the projection of $\mathbb{R}^{n}$ onto its first $k$ coordinates, that is, $\xi\left(x_{1}, \ldots, x_{n}\right)=\left(x_{1}, \ldots, x_{k}\right)$.

In the following, we state a new averaging theorem by Buică et al. in [28].

Theorem 3 (see [28]). Let $\beta: \bar{V} \rightarrow \mathbb{R}^{n-k}$ be a $C^{2}$ function, where $V$ is an open and bounded subset of $\mathbb{R}^{k}$. We assume that

(i) $\mathscr{Z}=\left\{z_{\alpha}=(\alpha, \beta(\alpha)), \alpha \in \bar{V}\right\} \subset \Omega$ and that for each $z_{\alpha} \in \mathscr{Z}$ the solution $X\left(t, z_{\alpha}\right)$ of (28) is T-periodic;

(ii) for each $z_{\alpha} \in \mathscr{Z}$ there is a fundamental matrix $M_{z_{\alpha}}(t)$ of (29) such that the matrix $M_{z_{\alpha}}^{-1}(0)-M_{z_{\alpha}}^{-1}(T)$ has in the upper right corner the $k \times(n-k)$ zero matrix, and in the lower right corner $a(n-k) \times(n-k)$ matrix $\Delta_{\alpha}$ with $\operatorname{det}\left(\Delta_{\alpha}\right) \neq 0$.

We consider the function $\mathscr{G}: \bar{V} \rightarrow \mathbb{R}^{k}$ :

$$
\mathscr{G}(\alpha)=\xi\left(\int_{0}^{T} M_{z_{\alpha}}^{-1}(t) G_{1}\left(t, X\left(t, z_{\alpha}\right)\right) \mathrm{d} t\right)
$$

If there exists $a \in V$ with $\mathscr{G}(a)=0$ and $\operatorname{det}((\mathrm{d} \mathscr{G} / \mathrm{d} \alpha)(a)) \neq 0$, then there is a T-periodic solution $X(t, \varepsilon)$ of system (27) such that $X(0, \varepsilon) \rightarrow z_{a}$ as $\varepsilon \rightarrow 0$.

\section{Proofs of Theorems 1 and 2}

In this section, we will show how to apply Theorem 3 given in Section 3 to prove our main theorems. The key step is to find a bounded and open set $V$, where there exist simple zeroes of the associated averaged system. We firstly prove the existence and multiplicity of periodic solutions for system (11), thereby for system (9), and then using the orthogonal transformation (8) return periodic solutions to the desired FPU system (5).

4.1. The Proof of Theorem 1. In order to apply Theorem 3, we rewrite system (11) in the following form:

$$
\dot{X}(t)=G_{0}(t, X(t))+\varepsilon G_{1}(t, X(t))+\mathcal{O}\left(\epsilon^{2}\right),
$$

where

$$
\begin{aligned}
& X=\left(\begin{array}{c}
\bar{q}_{1} \\
\bar{p}_{1} \\
\bar{q}_{2} \\
\bar{p}_{2} \\
\bar{q}_{3} \\
\bar{p}_{3}
\end{array}\right) \\
& G_{0}(t, X)=\left(\begin{array}{c}
\bar{p}_{1} \\
-4 \bar{q}_{1} \\
\bar{p}_{2} \\
-2 \bar{q}_{2} \\
\bar{p}_{3} \\
-2 \bar{q}_{3}
\end{array}\right), \\
& G_{1}(t, X)=\left(\begin{array}{c}
F_{1}\left(t, \bar{q}_{1}, \bar{p}_{1}, \bar{q}_{2}, \bar{p}_{2}, \bar{q}_{3}, \bar{p}_{3}\right) \\
0 \\
F_{2}\left(t, \bar{q}_{1}, \bar{p}_{1}, \bar{q}_{2}, \bar{p}_{2}, \bar{q}_{3}, \bar{p}_{3}\right) \\
0 \\
F_{3}\left(t, \bar{q}_{1}, \bar{p}_{1}, \bar{q}_{2}, \bar{p}_{2}, \bar{q}_{3}, \bar{p}_{3}\right)
\end{array}\right) .
\end{aligned}
$$

For the particular case of the differential system (27), we describe the different conditions which appear in the statement of Theorem 3. Therefore, we have that $n=6, k=2$, and the set $\Omega=\mathbb{R}^{6}$. Let $r_{1}>0$ be arbitrarily small and let $r_{2}>0$ be arbitrarily large. Define an open and bounded subset $V$ on the projection plane $\bar{q}_{1} O \bar{p}_{1}$ by

V

$$
=\left\{\alpha \in \mathbb{R}^{2}: \alpha=\left(\bar{q}_{10}, \bar{p}_{10}\right), r_{1}<\sqrt{\bar{q}_{10}^{2}+\bar{p}_{10}^{2}}<r_{2}\right\} .
$$

The function $\beta: \bar{V} \rightarrow \mathbb{R}^{4}$ is taken by $\beta(\alpha) \equiv(0,0,0,0)$. Obviously, $\beta$ is of class $C^{2}$. We define the set $\mathscr{Z}$ by

$$
\begin{aligned}
\mathscr{Z} & =\left\{z_{\alpha}=(\alpha, \beta(\alpha)): \alpha \in \bar{V}\right\}=\left\{\left(\bar{q}_{10}, \bar{p}_{10}, 0,0,0,0\right)\right. \\
& \left.\in \mathbb{R}^{6}: r_{1} \leq \sqrt{\bar{q}_{10}^{2}+\bar{p}_{10}^{2}} \leq r_{2}\right\} .
\end{aligned}
$$

As the origin is a periodic solution with arbitrary period of the unperturbed system, the aim of defining $r_{1}$ is to exclude the equilibrium point $(0,0,0,0,0,0)$. Obviously, for each $z_{\alpha} \epsilon$ $\mathscr{Z}$ and $\epsilon=0$, the solution $X\left(t, z_{\alpha}\right)$ of (31) with the initial value $X\left(0, z_{\alpha}\right)=z_{\alpha}$ is $\pi$-periodic with respect to $t$. In fact, every solution of (31) with $\epsilon=0$ starting from $\mathscr{Z}$ can be written by coordinates as $X\left(t, z_{\alpha}\right)=\left(\bar{q}_{1}\left(t, z_{\alpha}\right), \bar{p}_{1}\left(t, z_{\alpha}\right), \bar{q}_{2}(t\right.$, $\left.\left.z_{\alpha}\right), \bar{p}_{2}\left(t, z_{\alpha}\right), \bar{q}_{3}\left(t, z_{\alpha}\right), \bar{p}_{3}\left(t, z_{\alpha}\right)\right)$, where

$$
\begin{aligned}
& \bar{q}_{1}(t)=\bar{q}_{10} \cos (2 t)+\frac{1}{2} \bar{p}_{10} \sin (2 t), \\
& \bar{p}_{1}(t)=\bar{p}_{10} \cos (2 t)-2 \bar{q}_{10} \sin (2 t),
\end{aligned}
$$




$$
\begin{aligned}
& \bar{q}_{2}(t)=0, \\
& \bar{p}_{2}(t)=0, \\
& \bar{q}_{3}(t)=0, \\
& \bar{p}_{3}(t)=0 .
\end{aligned}
$$

Along the periodic solution $X\left(t, z_{\alpha}\right)$, the variational equation of the unperturbed system is given by

$$
\dot{Y}(t)=D_{X} G_{0}\left(t, X\left(t, z_{\alpha}\right)\right) Y(t),
$$

where $Y(t)$ is a $6 \times 6$ matrix function. Now we present the linearization matrix of the unperturbed system along the periodic solution by

$$
D_{X} G_{0}\left(t, X\left(t, z_{\alpha}\right)\right)=\left(\begin{array}{cccccc}
0 & 1 & 0 & 0 & 0 & 0 \\
-4 & 0 & 0 & 0 & 0 & 0 \\
0 & 0 & 0 & 1 & 0 & 0 \\
0 & 0 & -2 & 0 & 0 & 0 \\
0 & 0 & 0 & 0 & 0 & 1 \\
0 & 0 & 0 & 0 & -2 & 0
\end{array}\right) \text {. }
$$

The fundamental matrix $M_{z_{\alpha}}(t)$ of the variational equation (36), where $M_{z_{\alpha}}(0)$ is the identity matrix, adopts the simple following form:

$$
M_{z_{\alpha}}(t)=\left(\begin{array}{ccc}
A(t) & 0 & 0 \\
0 & B(t) & 0 \\
0 & 0 & B(t)
\end{array}\right)
$$

with

$$
\begin{aligned}
& A(t)=\left(\begin{array}{cc}
\cos (2 t) & \frac{1}{2} \sin (2 t) \\
-2 \sin (2 t) & \cos (2 t)
\end{array}\right), \\
& B(t)=\left(\begin{array}{cc}
\cos (\sqrt{2} t) & \frac{1}{\sqrt{2}} \sin (\sqrt{2} t) \\
-\sqrt{2} \sin (\sqrt{2} t) & \cos (\sqrt{2} t)
\end{array}\right) .
\end{aligned}
$$

Notice that $M_{z_{\alpha}}(t)$ does not depend on the initial value $z_{\alpha}$ of periodic solution $X\left(t, z_{\alpha}\right)$. Therefore, we drop the subscript $z_{\alpha}$ and reset $M(t)=M_{z_{\alpha}}(t)$. Computing the matrix $M^{-1}(0)-$ $M^{-1}(\pi)$, we get

$$
\left(\begin{array}{cccccc}
0 & 0 & 0 & 0 & 0 & 0 \\
0 & 0 & 0 & 0 & 0 & 0 \\
0 & 0 & 1-\cos (\sqrt{2} \pi) & \frac{1}{\sqrt{2}} \sin (\sqrt{2} \pi) & 0 & 0 \\
0 & 0 & -\sqrt{2} \sin (\sqrt{2} \pi) & 1-\cos (\sqrt{2} \pi) & 0 & 0 \\
0 & 0 & 0 & 0 & 1-\cos (\sqrt{2} \pi) & \frac{1}{\sqrt{2}} \sin (\sqrt{2} \pi) \\
0 & 0 & 0 & 0 & -\sqrt{2} \sin (\sqrt{2} \pi) & 1-\cos (\sqrt{2} \pi)
\end{array}\right) .
$$

This matrix has in the upper right corner $2 \times 4$ null matrix and in the lower right corner a $4 \times 4$ matrix $\Delta_{\alpha}$ satisfying

$$
\operatorname{det}\left(\Delta_{\alpha}\right)=\left|\begin{array}{cccc}
1-\cos (\sqrt{2} \pi) & \frac{1}{\sqrt{2}} \sin (\sqrt{2} \pi) & 0 & 0 \\
-\sqrt{2} \sin (\sqrt{2} \pi) & 1-\cos (\sqrt{2} \pi) & 0 & 0 \\
0 & 0 & 1-\cos (\sqrt{2} \pi) & \frac{1}{\sqrt{2}} \sin (\sqrt{2} \pi) \\
0 & 0 & -\sqrt{2} \sin (\sqrt{2} \pi) & 1-\cos (\sqrt{2} \pi)
\end{array}\right|=16 \sin ^{4}\left(\frac{\pi}{\sqrt{2}}\right) \neq 0 .
$$

Therefore, the assumption (ii) of Theorem 3 holds.

Following the notations of Theorem 3, we have

$$
\xi\left(\bar{q}_{1}, \bar{p}_{1}, \bar{q}_{2}, \bar{p}_{2}, \bar{q}_{3}, \bar{p}_{3}\right)=\left(\bar{q}_{1}, \bar{p}_{1}\right) .
$$

In the following, we will calculate the following function:

$$
\mathscr{G}\left(\bar{q}_{10}, \bar{p}_{10}\right)=\mathscr{G}(\alpha)
$$

$$
=\xi\left(\int_{0}^{T_{1}} M_{Z_{\alpha}}^{-1}(t) G_{1}\left(t, X\left(t, Z_{\alpha}\right)\right) \mathrm{d} t\right) .
$$

Let $\beta(t)=a+b \cos (4 t), \gamma(t)=\gamma_{0}$ with $a, b, \gamma_{0} \in \mathbb{R}$ and $\alpha(t)$, $\chi(t)$ be $C^{2}$ periodic function with the period $\pi$. Then it follows that $F_{1}\left(t, \bar{q}_{1}(t), \bar{p}_{1}(t), 0,0,0,0\right)$ is a $\pi$-periodic function only depending on $\beta(t)$ and $\gamma(t)$, which is not associated with the functions $\alpha(t)$ and $\chi(t)$. 
Therefore, we obtain

$$
\begin{aligned}
\mathscr{G}(\alpha) & =\left(\begin{array}{l}
\mathscr{F}_{1}\left(\bar{q}_{10}, \bar{p}_{10}\right) \\
\mathscr{F}_{2}\left(\bar{q}_{10}, \bar{p}_{10}\right)
\end{array}\right)=\left(\begin{array}{c}
-\int_{0}^{T_{1}} \frac{\sin (2 t)}{2} F_{1}\left(t, \bar{q}_{1}(t), \bar{p}_{1}(t), 0,0,0,0\right) \mathrm{d} t \\
\int_{0}^{T_{1}} \cos (2 t) F_{1}\left(t, \bar{q}_{1}(t), \bar{p}_{1}(t), 0,0,0,0\right) \mathrm{d} t
\end{array}\right) \\
& =\left(\begin{array}{c}
\frac{1}{256} \pi \bar{p}_{10}\left[8 \bar{p}_{10}^{2}\left(3 a-2 b+5 \gamma_{0} \bar{q}_{10}^{2}\right)+96 a \bar{q}_{10}^{2}+5 \gamma_{0} p_{10}^{4}+80 \gamma_{0} \bar{q}_{10}^{4}\right] \\
-\frac{1}{64} \pi \bar{q}_{10}\left(8 \bar{q}_{10}^{2}\left(12 a+8 b+5 \gamma_{0} \bar{p}_{10}^{2}\right)+24 a \bar{p}_{10}^{2}+5 \gamma_{0} \bar{p}_{10}^{4}+80 \gamma_{0} \bar{q}_{10}^{4}\right)
\end{array}\right) .
\end{aligned}
$$

Finally, we shall find the simple zeroes of the function $\mathscr{G}(\alpha)$ in the open and bounded subset $V \subset \mathbb{R}^{2}$. A straightforward computation shows that the solutions of $\mathscr{F}_{1}\left(\bar{q}_{10}, \bar{p}_{10}\right)=$ $0, \mathscr{F}_{2}\left(\bar{q}_{10}, \bar{p}_{10}\right)=0$ in $V$ are given by

$$
\begin{aligned}
\alpha_{1} & =(0,0), \\
\alpha_{2,3} & =\left( \pm \sqrt{\frac{-2(3 a+2 b)}{5 \gamma_{0}}}, 0\right), \\
\alpha_{4,5} & =\left(0, \pm 2 \sqrt{\frac{2(2 b-3 a)}{5 \gamma_{0}}}\right),
\end{aligned}
$$

whenever the radical expressions of (45) are well defined. We leave out of account the solution $\alpha_{1}$, since it may be corresponding to the trivial periodic solution, zero solution. Notice that $\alpha_{2,3}$ are well defined provided $(3 a+2 b) \gamma_{0}<0$; while $\alpha_{4,5}$ are well defined provided $(2 b-3 a) \gamma_{0}>0$. The Jacobian determinants are given by

$$
\begin{aligned}
& \operatorname{det}\left(\mathscr{G}_{\alpha}^{\prime}\left(\alpha_{2,3}\right)\right)=\frac{\pi^{2} b(3 a+2 b)^{3}}{25 \gamma_{0}^{2}}, \\
& \operatorname{det}\left(\mathscr{G}_{\alpha}^{\prime}\left(\alpha_{4,5}\right)\right)=-\frac{\pi^{2} b(3 a-2 b)^{3}}{25 \gamma_{0}^{2}},
\end{aligned}
$$

which means that $\left|G_{\alpha}^{\prime}\left(\alpha_{i}\right)\right| \neq 0(i=2, \ldots, 5)$ if $b \neq 0$, $(2 b-3 a) \gamma_{0}<0$ or $(3 a+2 b) \gamma_{0}>0$. Therefore, $\alpha_{i},(i=2, \ldots$, 5) are simple zeroes of the function $\mathscr{G}(\alpha)$. By Theorem 3 , there exist $\pi$-periodic solutions $X_{i}(t, \varepsilon)=\left(\bar{q}_{1 i}(t, \epsilon), \bar{p}_{1 i}(t\right.$, $\left.\epsilon), \bar{q}_{2 i}(t, \epsilon), \bar{p}_{2 i}(t, \epsilon), \bar{q}_{3 i}(t, \epsilon), \bar{p}_{3 i}(t, \epsilon)\right)$ of system (31) in different cases such that

$$
X_{i}(t, \varepsilon) \longrightarrow\left(\alpha_{i}, 0,0,0,0\right) \quad \text { as } \varepsilon \longrightarrow 0 .
$$

Note that, in our setting in Section 2 , we have already put $\bar{q}_{4}=\bar{p}_{4}=0$. Taking the inverse transformation of (8), we have

$$
\begin{aligned}
& q_{1 i}(t, \epsilon)=-\frac{1}{2} \bar{q}_{1 i}(t, \epsilon)-\frac{1}{\sqrt{2}} \bar{q}_{3 i}(t, \epsilon), \\
& p_{1 i}(t, \epsilon)=-\frac{1}{2} \bar{p}_{1 i}(t, \epsilon)-\frac{1}{\sqrt{2}} \bar{p}_{3 i}(t, \epsilon), \\
& q_{2 i}(t, \epsilon)=\frac{1}{2} \bar{q}_{1 i}(t, \epsilon)-\frac{1}{\sqrt{2}} \bar{q}_{2 i}(t, \epsilon),
\end{aligned}
$$

$$
\begin{aligned}
& p_{2 i}(t, \epsilon)=\frac{1}{2} \bar{p}_{1 i}(t, \epsilon)-\frac{1}{\sqrt{2}} \bar{p}_{2 i}(t, \epsilon), \\
& q_{3 i}(t, \epsilon)=-\frac{1}{2} \bar{q}_{1 i}(t, \epsilon)+\frac{1}{\sqrt{2}} \bar{q}_{3 i}(t, \epsilon), \\
& p_{3 i}(t, \epsilon)=-\frac{1}{2} \bar{p}_{1 i}(t, \epsilon)+\frac{1}{\sqrt{2}} \bar{p}_{3 i}(t, \epsilon), \\
& q_{4 i}(t, \epsilon)=\frac{1}{2} \bar{q}_{1 i}(t, \epsilon)+\frac{1}{\sqrt{2}} \bar{q}_{2 i}(t, \epsilon), \\
& p_{4 i}(t, \epsilon)=\frac{1}{2} \bar{p}_{1 i}(t, \epsilon)+\frac{1}{\sqrt{2}} \bar{p}_{2 i}(t, \epsilon) .
\end{aligned}
$$

Therefore, we get that, for $i=2,3$,

$$
\begin{aligned}
& \lim _{\epsilon \rightarrow 0}\left(q_{1 i}(0, \epsilon), p_{1 i}(0, \epsilon), q_{2 i}(0, \epsilon), p_{2 i}(0, \epsilon), q_{3 i}(0, \epsilon),\right. \\
& \left.p_{3 i}(0, \epsilon), q_{4 i}(0, \epsilon), p_{4 i}(0, \epsilon)\right)=\left(\mp \sqrt{\frac{-(3 a+2 b)}{10 \gamma_{0}}}, 0,\right. \\
& \pm \sqrt{\frac{-(3 a+2 b)}{10 \gamma_{0}}}, 0, \mp \sqrt{\frac{-(3 a+2 b)}{10 \gamma_{0}}}, 0, \\
& \left. \pm \sqrt{\frac{-(3 a+2 b)}{10 \gamma_{0}}}, 0\right)
\end{aligned}
$$

and, for $i=4,5$,

$$
\begin{aligned}
& \lim _{\epsilon \rightarrow 0}\left(q_{1 i}(0, \epsilon), p_{1 i}(0, \epsilon), q_{2 i}(0, \epsilon), p_{2 i}(0, \epsilon), q_{3 i}(0, \epsilon),\right. \\
& \left.p_{3 i}(0, \epsilon), q_{4 i}(0, \epsilon), p_{4 i}(0, \epsilon)\right)=\left(0, \mp \sqrt{\frac{2(2 b-3 a)}{5 \gamma_{0}}},\right. \\
& 0, \pm \sqrt{\frac{2(2 b-3 a)}{5 \gamma_{0}}}, 0, \mp \sqrt{\frac{2(2 b-3 a)}{5 \gamma_{0}}}, 0, \\
& \left. \pm \sqrt{\frac{2(2 b-3 a)}{5 \gamma_{0}}}\right) .
\end{aligned}
$$

Thus we complete the proof of Theorem 1. 
4.2. The Proof of Theorem 2. Let

$$
\begin{aligned}
& X=\left(\begin{array}{c}
\bar{q}_{2} \\
\bar{p}_{2} \\
\bar{q}_{3} \\
\bar{p}_{3} \\
\bar{q}_{1} \\
\bar{p}_{1}
\end{array}\right), \\
& G_{0}(t, X)=\left(\begin{array}{c}
\bar{p}_{2} \\
-2 \bar{q}_{2} \\
\bar{p}_{3} \\
-2 \bar{q}_{3} \\
\bar{p}_{1} \\
-4 \bar{q}_{1}
\end{array}\right), \\
& G_{1}(t, X)=\left(\begin{array}{c}
F_{2}\left(t, \bar{q}_{1}, \bar{p}_{1}, \bar{q}_{2}, \bar{p}_{2}, \bar{q}_{3}, \bar{p}_{3}\right) \\
0 \\
F_{3}\left(t, \bar{q}_{1}, \bar{p}_{1}, \bar{q}_{2}, \bar{p}_{2}, \bar{q}_{3}, \bar{p}_{3}\right) \\
0 \\
F_{1}\left(t, \bar{q}_{1}, \bar{p}_{1}, \bar{q}_{2}, \bar{p}_{2}, \bar{q}_{3}, \bar{p}_{3}\right)
\end{array}\right) .
\end{aligned}
$$

Then system (11) has the following form:

$$
\dot{X}(t)=G_{0}(t, X(t))+\varepsilon G_{1}(t, X(t))+\mathcal{O}\left(\epsilon^{2}\right) .
$$

Following the notations in Theorem 3, we have that $n=6$, $k=4$, and the set $\Omega=\mathbb{R}^{6}$. Let $r_{1}>0$ be arbitrarily small and let $r_{2}>0$ be arbitrarily large. Define an open and bounded subset $V$ on the projection plane $\bar{q}_{2} \bar{q}_{3} O \bar{p}_{2} \bar{p}_{3}$ by

$$
\begin{aligned}
V & =\left\{\alpha=\left(\bar{q}_{20}, \bar{p}_{20}, \bar{q}_{30}, \bar{p}_{30}\right) \in \mathbb{R}^{4}: r_{1}\right. \\
& \left.<\sqrt{\bar{q}_{20}^{2}+\bar{p}_{20}^{2}+\bar{q}_{30}^{2}+\bar{p}_{30}^{2}}<r_{2}\right\} .
\end{aligned}
$$

The function $\beta: \bar{V} \rightarrow \mathbb{R}^{2}$ is taken by $\beta(\alpha) \equiv(0,0)$. Consequently, we define the set $\mathscr{Z}$ by

$$
\begin{aligned}
\mathscr{E} & =\left\{z_{\alpha}=(\alpha, \beta(\alpha)): \alpha \in \bar{V}\right\} \\
& =\left\{\left(\bar{q}_{20}, \bar{p}_{20}, \bar{q}_{30}, \bar{p}_{30}, 0,0\right) \in \mathbb{R}^{6}: r_{1}\right. \\
& \left.\leq \sqrt{\bar{q}_{20}^{2}+\bar{p}_{20}^{2}+\bar{q}_{30}^{2}+\bar{p}_{30}^{2}} \leq r_{2}\right\} .
\end{aligned}
$$

Obviously, for each $z_{\alpha} \in \mathscr{Z}$ and $\epsilon=0$, the solution $X\left(t, z_{\alpha}\right)$ of (52) with the initial value $X\left(0, z_{\alpha}\right)=z_{\alpha}$ is $\sqrt{2} \pi$-periodic with respect to $t$. In fact, every solution $X\left(t, z_{\alpha}\right)$ of (52) with $\epsilon=0$ starting from $\mathscr{Z}$ is given by

$$
\begin{aligned}
& \bar{q}_{2}(t)=\bar{q}_{20} \cos (\sqrt{2} t)+\frac{1}{\sqrt{2}} \bar{p}_{20} \sin (\sqrt{2} t), \\
& \bar{p}_{2}(t)=\bar{p}_{20} \cos (\sqrt{2} t)-\sqrt{2} \bar{q}_{20} \sin (\sqrt{2} t),
\end{aligned}
$$

$$
\begin{aligned}
& \bar{q}_{3}(t)=\bar{q}_{30} \cos (\sqrt{2} t)+\frac{1}{\sqrt{2}} \bar{p}_{30} \sin (\sqrt{2} t), \\
& \bar{p}_{3}(t)=\bar{p}_{30} \cos (\sqrt{2} t)-\sqrt{2} \bar{q}_{30} \sin (\sqrt{2} t), \\
& \bar{q}_{1}(t)=0, \\
& \bar{p}_{1}(t)=0 .
\end{aligned}
$$

Accordingly, the variational equation of the unperturbed system,

$$
\dot{X}(t)=G_{0}(t, X(t)),
$$

along the periodic solution $X\left(t, z_{\alpha}\right)$, is given by

$$
\begin{aligned}
\dot{Y}(t) & =D_{X} G_{0}\left(t, X\left(t, z_{\alpha}\right)\right) Y(t) \\
& =\left(\begin{array}{cccccc}
0 & 1 & 0 & 0 & 0 & 0 \\
-2 & 0 & 0 & 0 & 0 & 0 \\
0 & 0 & 0 & 1 & 0 & 0 \\
0 & 0 & -2 & 0 & 0 & 0 \\
0 & 0 & 0 & 0 & 0 & 1 \\
0 & 0 & 0 & 0 & -4 & 0
\end{array}\right) Y(t),
\end{aligned}
$$

where $Y(t)$ is a $6 \times 6$ matrix function. The fundamental matrix $M_{z_{\alpha}}(t)$ of (57), which satisfies that $M_{z_{\alpha}}(0)$ is the identity matrix, adopts the simple following form:

$$
M_{z_{\alpha}}(t)=\left(\begin{array}{ccc}
A(t) & 0 & 0 \\
0 & A(t) & 0 \\
0 & 0 & B(t)
\end{array}\right)
$$

with

$$
\begin{aligned}
& A(t)=\left(\begin{array}{cc}
\cos (\sqrt{2} t) & \frac{1}{\sqrt{2}} \sin (\sqrt{2} t) \\
-\sqrt{2} \sin (\sqrt{2} t) & \cos (\sqrt{2} t)
\end{array}\right), \\
& B(t)=\left(\begin{array}{cc}
\cos (2 t) & \frac{1}{2} \sin (2 t) \\
-2 \sin (2 t) & \cos (2 t)
\end{array}\right) .
\end{aligned}
$$

Note that $M_{z_{\alpha}}(t)$ does not depend on the initial value $z_{\alpha}$ of periodic solution $X\left(t, z_{\alpha}\right)$. Therefore, we drop the subscript $z_{\alpha}$ and reset $M(t)=M_{z_{\alpha}}(t)$. With a direct calculation, we get

$$
\begin{aligned}
& M^{-1}(0)-M^{-1}(\sqrt{2} \pi) \\
& =\left(\begin{array}{cccccc}
0 & 0 & 0 & 0 & 0 & 0 \\
0 & 0 & 0 & 0 & 0 & 0 \\
0 & 0 & 0 & 0 & 0 & 0 \\
0 & 0 & 0 & 0 & 0 & 0 \\
0 & 0 & 0 & 0 & 1-\cos (2 \sqrt{2} \pi) & \frac{1}{2} \sin (2 \sqrt{2} \pi) \\
0 & 0 & 0 & 0 & -2 \sin (2 \sqrt{2} \pi) & 1-\cos (2 \sqrt{2} \pi)
\end{array}\right) .
\end{aligned}
$$


We observe that the matrix $M^{-1}(0)-M^{-1}(\sqrt{2} \pi)$ has in the upper right corner the $4 \times 2$ null matrix and in the lower right corner a $2 \times 2$ matrix $\Delta_{\alpha}$ satisfying

$$
\begin{aligned}
\operatorname{det}\left(\Delta_{\alpha}\right) & =\left|\begin{array}{cc}
1-\cos (2 \sqrt{2} \pi) & \frac{1}{2} \sin (2 \sqrt{2} \pi) \\
-2 \sin (2 \sqrt{2} \pi) & 1-\cos (2 \sqrt{2} \pi)
\end{array}\right| \\
& =4 \sin ^{2}(\sqrt{2} \pi) \neq 0 .
\end{aligned}
$$

Therefore, assumptions (i) and (ii) of Theorem 3 hold.
Following the notation of Theorem 3, let us define the projection $\xi: \mathbb{R}^{6} \rightarrow \mathbb{R}^{4}$ by

$$
\xi\left(\bar{q}_{2}, \bar{p}_{2}, \bar{q}_{3}, \bar{p}_{3}, \bar{q}_{1}, \bar{p}_{1}\right)=\left(\bar{q}_{2}, \bar{p}_{2}, \bar{q}_{3}, \bar{p}_{3}\right) .
$$

Since the functions $\beta(t)=b \cos (2 \sqrt{2} t), \gamma(t)=\gamma_{0}, b, \gamma_{0} \in \mathbb{R}$, $\alpha(t), \chi(t)$ are all $\sqrt{2} \pi$-periodic and of class $C^{2}$, we know that $F_{2}, F_{3}$ are also $\sqrt{2} \pi$-periodic functions which only depends on $\beta(t)$ and $\gamma(t)$. By a straightforward computation, we obtain that

$$
\begin{aligned}
& \mathscr{G}(\alpha)=\xi\left(\int_{0}^{\sqrt{2} \pi} M_{Z_{\alpha}}^{-1}(t) G_{1}\left(t, X\left(t, z_{\alpha}\right)\right) \mathrm{d} t\right)=\left(\mathscr{G}_{1}, \mathscr{G}_{2}, \mathscr{G}_{3}, \mathscr{G}_{4}\right)^{T}=\left(\begin{array}{c}
-\int_{0}^{\sqrt{2} \pi} \frac{\sin (\sqrt{2} t)}{\sqrt{2}} F_{2}\left(t, 0,0, \bar{q}_{2}(t), \bar{p}_{2}(t), \bar{q}_{3}(t), \bar{p}_{3}(t)\right) \mathrm{d} t \\
\int_{0}^{\sqrt{2} \pi} \cos (\sqrt{2} t) F_{2}\left(t, 0,0, \bar{q}_{2}(t), \bar{p}_{2}(t), \bar{q}_{3}(t), \bar{p}_{3}(t)\right) \mathrm{d} t \\
-\int_{0}^{\sqrt{2} \pi} \frac{\sin (\sqrt{2} t)}{\sqrt{2}} F_{3}\left(t, 0,0, \bar{q}_{2}(t), \bar{p}_{2}(t), \bar{q}_{3}(t), \bar{p}_{3}(t)\right) \mathrm{d} t \\
\int_{0}^{\sqrt{2} \pi} \cos (\sqrt{2} t) F_{3}\left(t, 0,0, \bar{q}_{2}(t), \bar{p}_{2}(t), \bar{q}_{3}(t), \bar{p}_{3}(t)\right) \mathrm{d} t
\end{array}\right) \\
& =\frac{\pi}{128 \sqrt{2}} \\
& \cdot\left(\begin{array}{c}
5 \gamma p_{20}^{5}+120 \gamma p_{p_{0}}^{2} p_{30} q_{20} q_{30}+40 \gamma p_{30} q_{20} q_{30}\left(p_{30}^{2}+2\left(q_{20}^{2}+q_{30}^{2}\right)\right)+2 p_{20}^{3}\left(-8 b+25 \gamma p_{30}^{2}+10 \gamma\left(q_{20}^{2}+q_{30}^{2}\right)\right)+p_{20}\left(25 \gamma p_{30}^{4}+20 \gamma\left(q_{20}^{4}+6 q_{20}^{2} q_{30}^{2}+q_{30}^{4}\right)+12 p_{30}^{2}\left(-4 b+5 \gamma\left(q_{20}^{2}+q_{30}^{2}\right)\right)\right) \\
2\left(-5 \gamma_{0}\left(\bar{p}_{20}^{4}+6 \bar{p}_{20}^{2} \bar{p}_{30}^{2}+\bar{p}_{30}^{4}\right) \bar{q}_{20}-4\left(8 b+5 \gamma_{0}\left(\bar{p}_{20}^{2}+\bar{p}_{30}^{2}\right)\right) \bar{q}_{20}-20 \gamma_{0} \bar{q}_{20}-20 \gamma_{0} \bar{p}_{20} \bar{p}_{30}\left(\bar{p}_{20}^{2}+\bar{p}_{30}^{2}+6 \bar{q}_{20}^{2}\right) \bar{q}_{30}-4 \bar{q}_{20}\left(24 b+15 \gamma_{0}\left(\bar{p}_{20}^{2}+p_{30}^{2}\right)+50 \gamma_{0} \bar{q}_{20}^{2}\right) \bar{q}_{30}^{2}-40 \gamma_{0} \bar{p}_{20} \bar{p}_{3} \bar{q}_{30}-100 \gamma_{0} \bar{q}_{20} \bar{q}_{30}^{4}\right) \\
25 \gamma_{0} \bar{p}_{20}^{4} \bar{p}_{30}+40 \gamma_{0} \bar{p}_{20}^{3} \bar{q}_{20} \bar{q}_{30}+40 \gamma_{0} \bar{p}_{20} \bar{q}_{20} \bar{q}_{30}\left(3 \bar{p}_{30}^{2}+2\left(\bar{q}_{20}^{2}+\bar{q}_{30}^{2}\right)\right)+2 \bar{p}_{20} \bar{p}_{30}\left(-24 b+5 \gamma_{0}\left(5 \bar{p}_{30}^{2}+6\left(\bar{q}_{20}^{2}+\bar{q}_{30}^{2}\right)\right)\right)+\bar{p}_{30}\left(5 \gamma_{0} \bar{p}_{30}^{4}+20 \gamma_{0}\left(\bar{q}_{20}^{4}+6 \bar{q}_{20}^{2} \bar{q}_{30}^{2}+\bar{q}_{30}^{4}\right)+4 \bar{p}_{30}^{2}\left(-4 b+5 \gamma_{0}\left(\bar{q}_{20}^{2}+\bar{q}_{30}^{2}\right)\right)\right) \\
2\left(-20 \gamma_{0} \bar{p}_{20} \bar{p}_{30} \bar{q}_{20}\left(\bar{p}_{20}^{2}+\bar{p}_{30}^{2}+2 \bar{q}_{20}^{2}\right)-\left(5 \gamma_{0}\left(\bar{p}_{20}^{4}+6 \bar{p}_{20}^{2} \bar{p}_{30}^{2}+\bar{p}_{30}^{4}\right)+12\left(8 b+5 \gamma_{0}\left(\bar{p}_{20}^{2}+\bar{p}_{30}^{2}\right)\right) \bar{q}_{20}^{2}+100 \gamma_{0} \bar{q}_{20} 2^{4}\right) \bar{q}_{30}-20 \gamma_{0} q_{30}^{5}-120 \gamma_{0} \bar{p}_{20} \bar{p}_{30} \bar{q}_{20} \bar{q}_{30}^{2}-4\left(8 b+5 \gamma_{0}\left(\bar{p}_{20}^{2}+\bar{p}_{30}^{2}+10 \bar{q}_{20}^{2}\right)\right) \bar{q}_{30}^{3}\right)
\end{array}\right)
\end{aligned}
$$

Now, we shall compute the simple zeroes of the function $\mathscr{G}(\alpha)$ in the open and bounded subset $V \subset \mathbb{R}^{4}$. A straightforward computation shows that the solutions of $\mathscr{G}(\alpha)=0$ in $V$ are given by

$$
\begin{aligned}
\alpha_{1} & =(0,0,0,0), \\
\alpha_{2,3} & =\left(0, \pm 4 \sqrt{\frac{b}{5 \gamma_{0}}}, 0,0\right), \\
\alpha_{4,5} & =\left(0,0,0, \pm 4 \sqrt{\frac{b}{5 \gamma_{0}}}\right),
\end{aligned}
$$

whenever the radical expressions (64) are well defined. Notice that $\alpha_{2,3,4,5}$ are well defined provided $b \gamma_{0}>0$. The associated Jacobian determinants are given by

$$
\operatorname{det}\left(\mathscr{G}_{\alpha}^{\prime}\left(\alpha_{2,3,4,5}\right)\right)=\frac{64 \pi^{4} b^{8}}{625 \gamma_{0}^{4}} .
$$

This means that $\left|\mathscr{G}_{\alpha}^{\prime}\left(\alpha_{i}\right)\right| \neq 0(i=2, \ldots, 5)$ for $b \gamma_{0}>0$. Therefore, $\alpha_{i}, i=2, \ldots, 5$, are simple zeroes. With the same arguments in the proof of Theorem 1, we can complete the proof of Theorem 2 by taking the inverse transformation of (8).

\section{Numerical Simulation}

In order to support our results, we perform some numerical simulations. Although we cannot find the exact periodic solutions, according to proposed theorems (see Theorems 1 and 2) and their proofs, the initial values of periodic solutions of (11) are near its simple zeroes. The differences between the simple zeroes and the initial values of corresponding periodic solutions are of one order $\varepsilon$.

First, we take $\epsilon=0.001$ and

$$
\begin{aligned}
& \alpha(t)=1+2 \sin (2 t), \\
& \beta(t)=1+\cos (4 t), \\
& \chi(t)=\cos (2 t), \\
& \gamma(t)=-\frac{1}{2} .
\end{aligned}
$$

By Theorem 1, we know that there exist four $\pi$-periodic solutions (since the conditions in (a) and (b) of Theorem 1 simultaneously hold):

$$
\begin{aligned}
& \left(q_{1}(t, \varepsilon), p_{1}(t, \varepsilon), q_{2}(t, \varepsilon), p_{2}(t, \varepsilon), q_{2}(t, \varepsilon), p_{3}(t, \varepsilon),\right. \\
& \left.q_{2}(t, \varepsilon), p_{4}(t, \varepsilon)\right)
\end{aligned}
$$

such that

$$
\begin{aligned}
& \lim _{\epsilon \rightarrow 0}\left(q_{1}(0, \epsilon), p_{1}(0, \epsilon), q_{2}(0, \epsilon), p_{2}(0, \epsilon), q_{3}(0, \epsilon),\right. \\
& \left.\quad p_{3}(0, \epsilon), q_{4}(0, \epsilon), p_{4}(0, \epsilon)\right)=(\mp 1,0, \pm 1,0, \mp 1,0, \\
& \quad \pm 1,0) . \\
& \text { or } \quad\left(0, \mp \frac{2}{\sqrt{5}}, 0, \pm \frac{2}{\sqrt{5}}, 0, \mp \frac{2}{\sqrt{5}}, 0, \pm \frac{2}{\sqrt{5}}\right)
\end{aligned}
$$



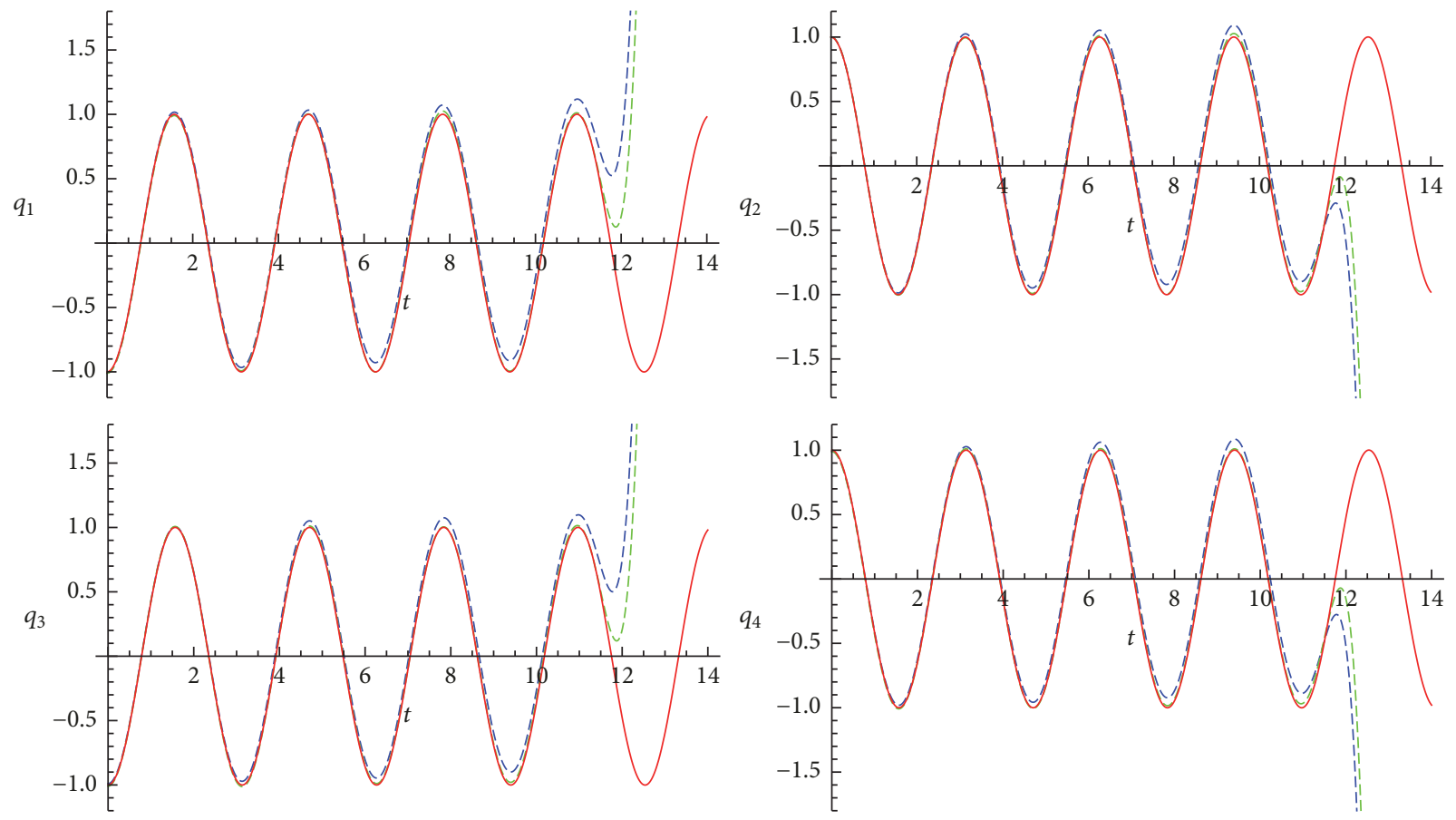

FIGURE 1: The curves of solutions of (5): the red curve is an approximate $\pi$-periodic solution starting from $P_{1}$, while the blue dashed curve and the green dashed curve are the reference solutions starting from $Q_{11}$ and $Q_{12}$, respectively.

To perform numerical simulations for FPU system (5), we take the initial values

$$
\begin{gathered}
P_{1}:(-1,0,1,0,-1,0,1,0), \\
P_{2}:(1,0,-1,0,1,0,-1,0)
\end{gathered}
$$

for the approximate $\pi$-periodic solutions and take the initial values

$$
\begin{gathered}
Q_{11}:(-1.01,-0.01,1,0.01,-1.01,0.02,0.99,-0.01), \\
Q_{12}:(-1.01,0.01,1,0.01,-0.99,0.01,0.99,0.01), \\
Q_{21}:(1.01,-0.01,-1,0.01,1.01,0.02,-0.99,-0.01), \\
Q_{22}:(1.01,0.01,-1,0.01,0.99,0.01,-0.99,0.01)
\end{gathered}
$$

for the reference solutions. We plot the curves of solutions by approximate calculation, which start from the different initial values $P_{i}, Q_{i j}, i, j=1,2$. As is shown in Figures 1 and 2 , the solution (red curve) starting from $P_{1}$ or $P_{2}$ is approximately $\pi$ periodic. We also can simulate the other $\pi$-periodic solutions starting from $(0, \mp 2 / \sqrt{5}, 0, \pm 2 / \sqrt{5}, 0, \mp 2 / \sqrt{5}, 0, \pm 2 / \sqrt{5})$, but we omit it here since there are no techniques.

Next, we take $\epsilon=0.001$ and

$$
\begin{aligned}
& \alpha(t)=1+\sin (\sqrt{2} t), \\
& \beta(t)=5 \cos (2 \sqrt{2} t), \\
& \chi(t)=\cos (\sqrt{2} t), \\
& \gamma(t)=2 .
\end{aligned}
$$

By Theorem 2, we know that there exist four $\sqrt{2} \pi$-periodic solutions:

$$
\begin{aligned}
& \left(q_{1}(t, \varepsilon), p_{1}(t, \varepsilon), q_{2}(t, \varepsilon), p_{2}(t, \varepsilon), q_{2}(t, \varepsilon), p_{3}(t, \varepsilon),\right. \\
& \left.\quad q_{2}(t, \varepsilon), p_{4}(t, \varepsilon)\right)
\end{aligned}
$$

such that

$$
\begin{aligned}
\lim _{\epsilon \rightarrow 0}\left(q_{1}(0, \epsilon), p_{1}(0, \epsilon), q_{2}(0, \epsilon), p_{2}(0, \epsilon), q_{3}(0, \epsilon),\right. \\
\left.p_{3}(0, \epsilon), q_{4}(0, \epsilon), p_{4}(0, \epsilon)\right)=(0,0,0, \mp 2,0,0,0, \pm 2)
\end{aligned}
$$

or $(0, \mp 2,0,0,0, \pm 2,0,0)$.

To demonstrate the correctness of our results, we will choose one of $\varepsilon$-limit points $P_{3}:(0,0,0,-2,0,0,0,2)$ of the exact periodic solutions and two reference points:

$$
\begin{gathered}
Q_{31}:(0.01,-0.01,0.01,-2.01,0.01,0.02,-0.01,2), \\
Q_{32}:(-0.01,0,0.01,-1.98,0.01,0.01,0.01,2.01)
\end{gathered}
$$

as the initial values to perform numerical simulations. As is shown in Figure 3, the solution (red curve) starting from $P_{3}$ is approximately $\sqrt{2} \pi$-periodic, while obviously the other solutions (the blue dashed curve and the green dashed curve) starting from $Q_{31}$ and $Q_{32}$ are not $\sqrt{2} \pi$-periodic.

\section{Conclusions and Further Discussions}

The paper studies the existence and multiplicity of periodic orbits of four-particle time-dependent FPU chains by averaging theory which is one of important methods in the area of 

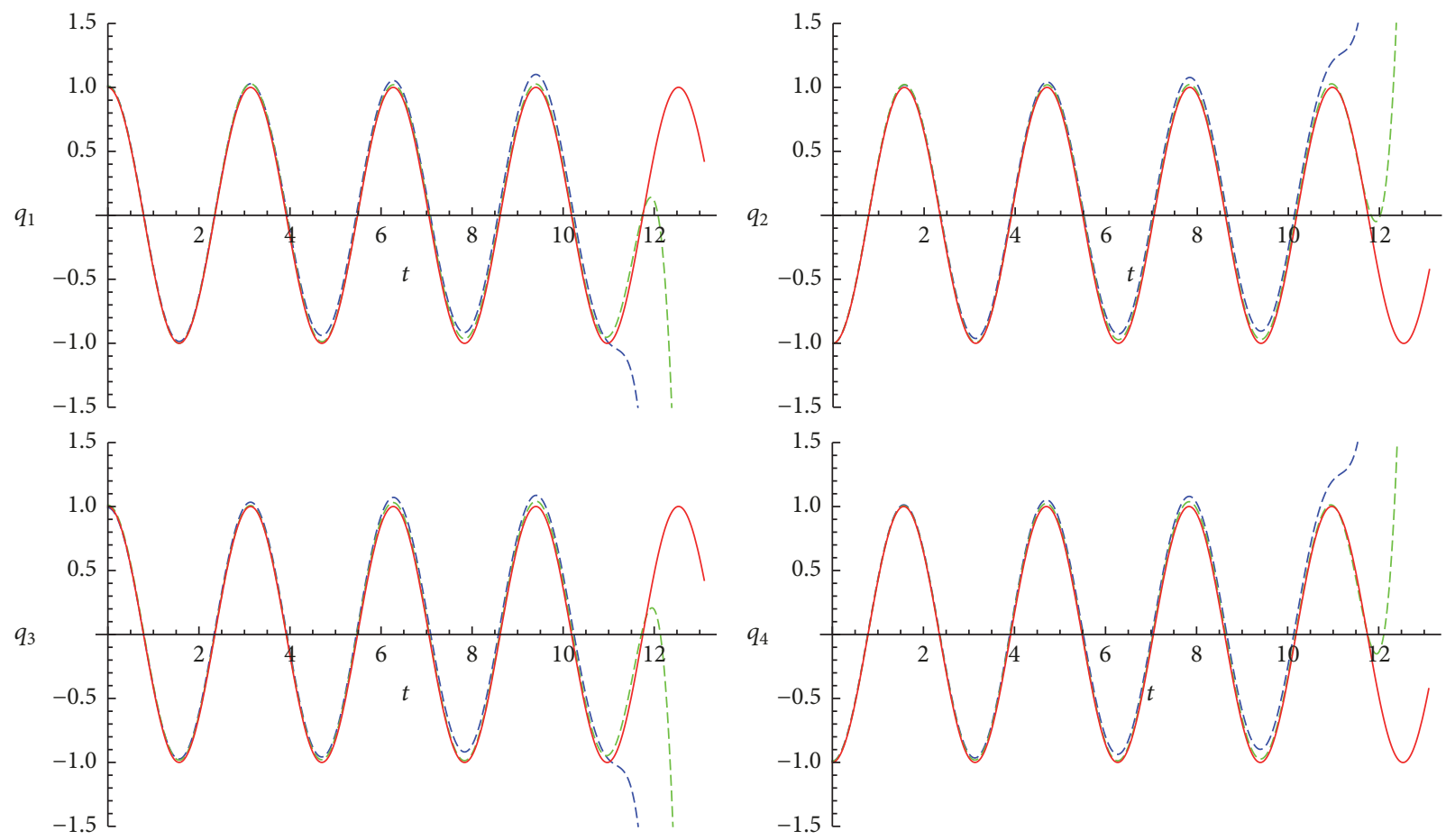

FIGURE 2: The curves of solutions of (5): the red curve is an approximate $\pi$-periodic solution starting from $P_{2}$, while the blue dashed curve and the green dashed curve are the reference solutions starting from $Q_{21}$ and $Q_{22}$, respectively.
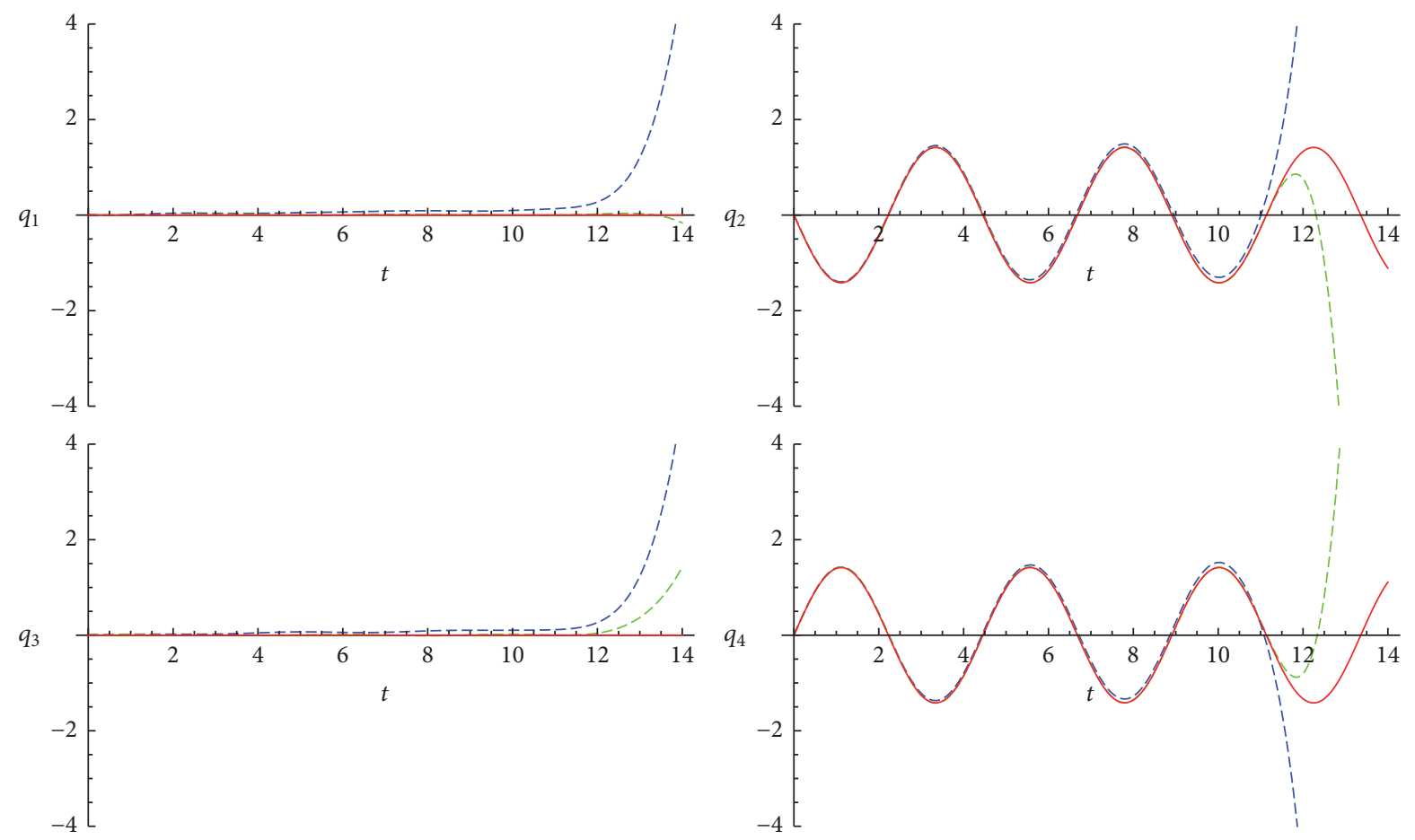

FIGURE 3: The curves of solutions of (5): the red curve is an approximate $\sqrt{2} \pi$-periodic solution starting from $P_{3}$, while the blue dashed curve and the green dashed curve are the reference solutions starting from $Q_{31}$ and $Q_{32}$, respectively. 
dynamical system. In this case, the classical averaging theory cannot be applied directly by reason of the unperturbed system with multiple irrational frequencies. Therefore, to overcome this difficulty, we apply a new result from the averaging theory as the main mathematical tool. Theorems 1 and 2 are our main results on the periodic orbits for fourdimensional time-dependent FPU chains. We conclude that four-particle time-dependent FPU system (5) has multiplicity nontrivial periodic solution.

Moreover, the method used in this paper is easy to apply for $N(N>4)$ particles FPU chains. Of course, with the increase of the dimension of system, the amount of calculation may increase much more. The method used for proving the existence and multiplicity of periodic orbits of fourparticle time-dependent FPU chains can be applied to other Hamiltonian systems with multiple irrational frequencies. We expect the numerical and theoretical results in the near future.

\section{Conflicts of Interest}

The authors declare that there are no conflicts of interest.

\section{Acknowledgments}

This work was supported in part by the National Natural Science Foundation of China (Grants nos. 11771105 and 11662001) and Guangxi Natural Science Foundation (Grants nos. 2017GXNSFFA198012 and 2015GXNSFGA139004).

\section{References}

[1] P. Poggi and S. Ruffo, "Exact solutions in the FPU oscillator chain," Physica D: Nonlinear Phenomena, vol. 103, no. 1-4, pp. 251-272, 1997.

[2] E. Fermi, J. Pasta, and S. Ulam, "Studies of nonlinear problems," Tech. Rep., Report LA-1940 2: 977-988, Los Alamos, 1955.

[3] D. K. Campbell, P. Rosenau, and G. M. Zaslavsky, "Introduction: The Fermi-Pasta-Ulam problem - The first fifty years," Chaos: An Interdisciplinary Journal of Nonlinear Science, vol. 15, no. 1, Article ID 015101, 2005.

[4] C. Giardinà and R. Livi, "Ergodic properties of microcanonical observables," Journal of Statistical Physics, vol. 91, no. 5-6, pp. 1027-1045, 1998.

[5] B. Rink, "Symmetry and resonance in periodic FPU chains," Communications in Mathematical Physics, vol. 218, no. 3, pp. 665-685, 2001.

[6] B. Rink and F. Verhulst, "Near-integrability of periodic FPUchains," Physica A: Statistical Mechanics and its Applications, vol. 285, no. 3, pp. 467-482, 2000.

[7] G. Benettin, H. Christodoulidi, and A. Ponno, "The FermiPasta-Ulam problem and its underlying integrable dynamics," Journal of Statistical Physics, vol. 152, no. 2, pp. 195-212, 2013.

[8] G. James, "Existence of breathers on FPU lattices," Comptes Rendus de $l$ Académie des Sciences - Series I - Mathematics (Compt Rendus Acad Sci Math), vol. 332, no. 6, pp. 581-586, 2001.

[9] X. Yuan, "Construction of quasi-periodic breathers via KAM technique," Communications in Mathematical Physics, vol. 226, no. 1, pp. 61-100, 2002.
[10] G. James and P. Noble, "Breathers on diatomic Fermi-PastaUlam lattices," Physica D: Nonlinear Phenomena, vol. 196, no. 1-2, pp. 124-171, 2004.

[11] C. Antonopoulos and T. Bountis, "Stability of simple periodic orbits and chaos in a Fermi-Pasta-Ulam lattice," Physical Review E: Statistical, Nonlinear, and Soft Matter Physics, vol. 73, no. 5, Article ID 056206, 056206, 8 pages, 2006.

[12] T. Bountis and H. Skokos, "FPU Recurrences and the Transition from Weak to Strong Chaos," in Complex Hamiltonian Dynamics, vol. 10 of Springer Series in Synergetics, pp. 133-164, Springer Berlin Heidelberg, Berlin, Heidelberg, 2012.

[13] T. Penati and S. Flach, "Tail resonances of Fermi-Pasta-Ulam q-breathers and their impact on the pathway to equipartition," Chaos: An Interdisciplinary Journal of Nonlinear Science, vol. 17, no. 2, Article ID 023102, 2007.

[14] S. Flach, M. V. Ivanchenko, O. I. Kanakov, and K. G. Mishagin, "Periodic orbits, localization in normal mode space, and the Fermi-Pasta-Ulam problem," American Journal of Physics, vol. 76, no. 4-5, pp. 453-459, 2008.

[15] S.-I. Sasa and T. S. Komatsu, "Thermodynamic entropy and excess information loss in dynamical systems with timedependent hamiltonians," Physical Review Letters, vol. 82, no. 5, pp. 912-915, 1999.

[16] G. Gallavotti, Ed., The Fermi-Pasta-Ulam problem: a status report, Springer, Berlin Heidelberg, 2007.

[17] D. Bambusi and A. Ponno, "On metastability in FPU," Communications in Mathematical Physics, vol. 264, no. 2, pp. 539-561, 2006.

[18] S. Flach and A. Ponno, "The Fermi-Pasta-Ulam problem: periodic orbits, normal forms and resonance overlap criteria," Physica D: Nonlinear Phenomena, vol. 237, no. 7, pp. 908-917, 2008.

[19] F. Martínez-Farías, P. Panayotaros, and A. Olvera, "Weakly nonlinear localization for a 1-D FPU chain with clustering zones," The European Physical Journal Special Topics, vol. 223, no. 13, pp. 2943-2952, 2014.

[20] J. Sun and S. Ma, "Nontrivial periodic motions for resonant type asymptotically linear lattice dynamical systems," Journal of Mathematical Analysis and Applications, vol. 417, no. 2, pp. 622634, 2014.

[21] J. A. Sanders and F. Verhulst, Averaging Methods in Nonlinear Dynamical Systems, vol. 59 of Applied Mathematical Sciences, Springer, New York, NY, USA, 1985.

[22] M. T. de Bustos, M. A. López, and R. Martínez, "On the periodic solutions of a linear chain of three identical atoms," in Nonlinear Dynamics, vol. 76, pp. 893-903, 2014.

[23] M. Kulesza and J. Llibre, "The 3-dimensional cored and logarithm potentials: periodic orbits," Journal of Mathematical Physics, vol. 55, no. 11, Article ID 112702, 2014.

[24] Q. Liu and D. Qian, "Modulated amplitude waves with nonzero phases in Bose-Einstein condensates," Journal of Mathematical Physics, vol. 52, no. 8, Article ID 082702, 2011.

[25] L. Jia, Q. Liu, and Z. Ma, "A good approximation of modulated amplitude waves in Bose-Einstein condensates," Communications in Nonlinear Science and Numerical Simulation, vol. 19, no. 8, pp. 2715-2723, 2014.

[26] Q. Liu and D. Qian, "Nonlinear dynamics of differential equations with attractive-repulsive singularities and small timedependent coefficients," Mathematical Methods in the Applied Sciences, vol. 36, no. 2, pp. 227-233, 2013.

[27] Q.-H. Liu, M.-Y. Xing, X.-X. Li, and C. Wang, "Unstable and exact periodic solutions of three-particles time-dependent FPU chains," Chinese Physics B, vol. 24, no. 12, Article ID 120401, 2015. 
[28] A. Buică, J.-P. Françoise, and J. Llibre, "Periodic solutions of nonlinear periodic differential systems with a small parameter," Communications on Pure and Applied Analysis, vol. 6, no. 1, pp. 103-111, 2007.

[29] F. Verhulst, Nonlinear Differential Equations and Dynamical Systems, Springer, Berlin, Heidelberg, Germany, 1996. 


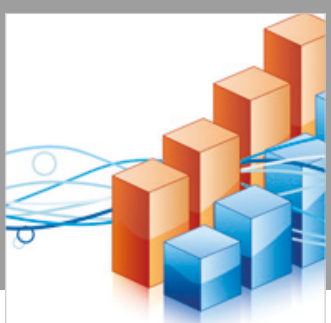

Advances in

Operations Research

\section{-n-m}
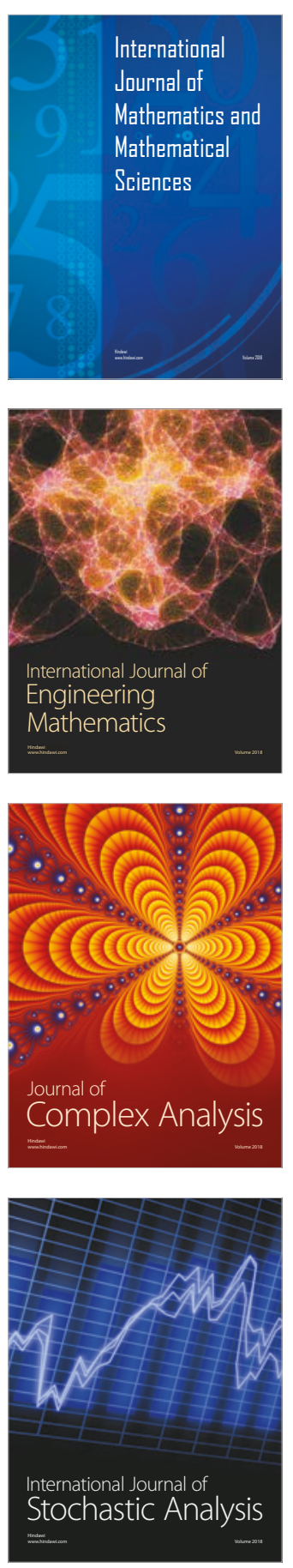
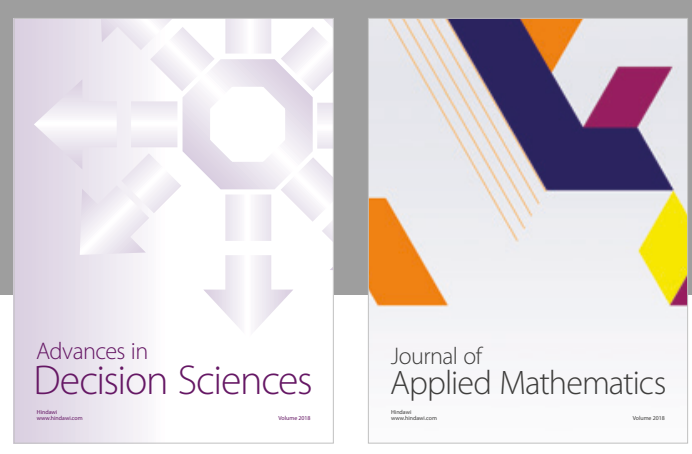

Journal of

Applied Mathematics
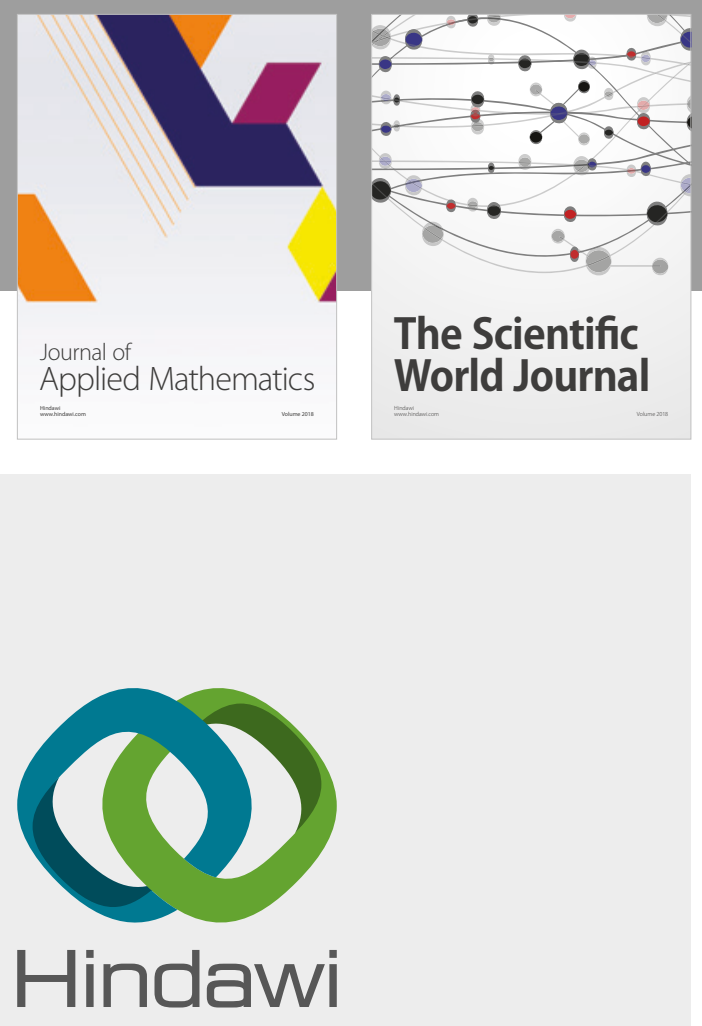

Submit your manuscripts at

www.hindawi.com

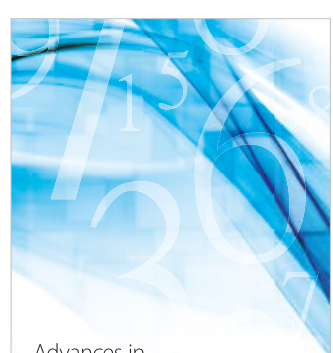

Advances in
Numerical Analysis
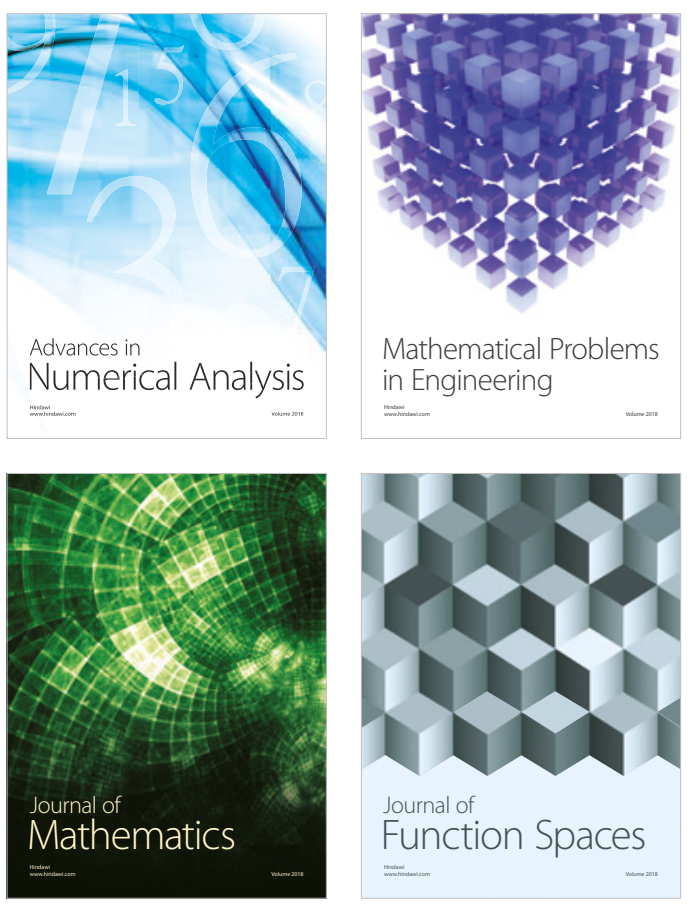

Mathematical Problems in Engineering

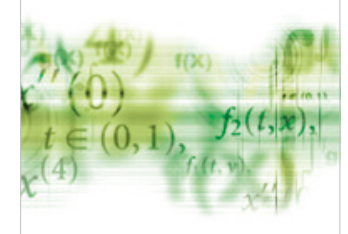

International Journal of

Differential Equations

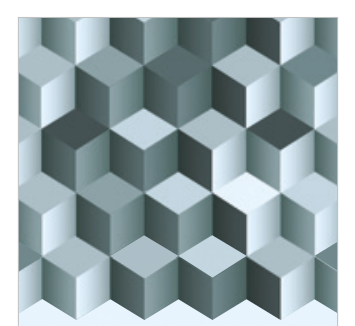

Journal of

Function Spaces

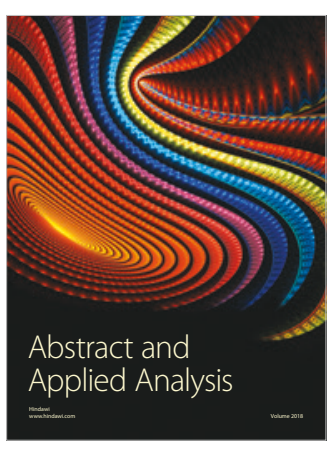

The Scientific

World Journal

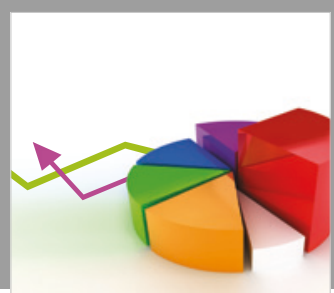

Journal of

Probability and Statistics
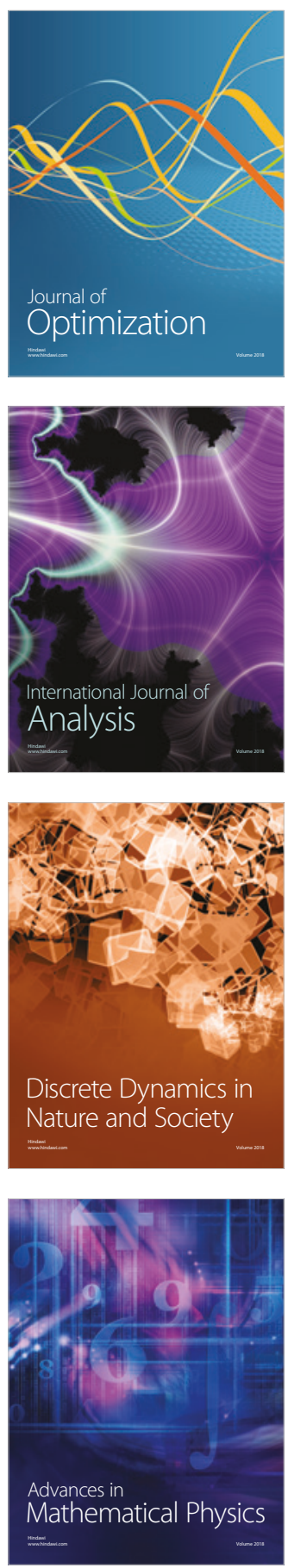